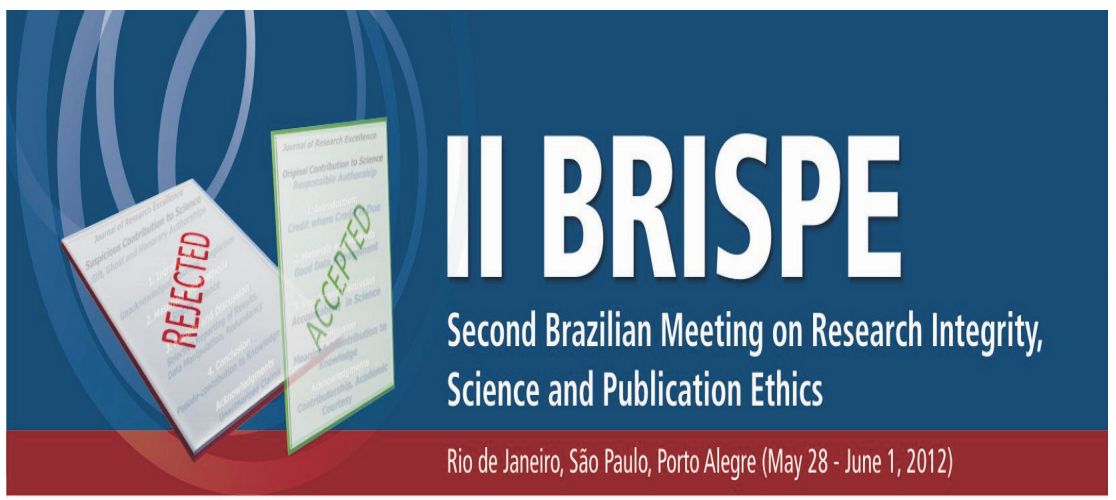

\title{
Declaração Conjunta sobre Integridade em Pesquisa do II Encontro Brasileiro de Integridade em Pesquisa, Ética na Ciência e em Publicações (II BRISPE), 28 Maio-01 de Junho de 2012
}

(Recomendações dos Membros Participantes do Grupo de Trabalho do II BRISPE)

O Brasil vem se tornando rapidamente uma potência científica e sua comunidade de pesquisa vem estabelecendo uma posição de liderança não apenas no cenário regional da ciência na América Latina, mas também no cenário internacional. Estabelecer essa liderança, entretanto, exige mais do que publicações científicas de alto impacto e pesquisadores qualificados. Embora essas características sejam tradicionalmente reconhecidas como marcas de excelência, elas têm pouco valor se não estiverem atrelada s a altos padrões de integridade científica [1,2]. Essa característica é mais do que evidente nos grandes desafios éticos que hoje se revelam nas discussões globais sobre novas tecnologias, mudanças climáticas, exploração dos recursos naturais, mas também sobre ciência e sociedade, proteção e compartilhamento de dados, sobre direitos autorais e propriedade intelectual $[3,4]$. Neste panorama global, as seguintes questões devem ser consideradas:

i. A responsabilização nas atividades científicas e a confiança pública nos resultados de pesquisa são hoje percebidas como parte dos desafios éticos mais atuais na ciência contemporânea, sobretudo num momento em que se inte nsificam os diálogos entre ciência e sociedade.

ii. A responsabilização nas atividades científicas e a confiança pública na ciência são hoje consideradas aspectos cruciais no âmbito da governança em ciência, tecnologia e inovação $(C, T \& I)$. Essa responsabilização está intimamente relacionada à promoção da integridade científica, como já sinalizado em documentos internacionais, como a Declaração de Cingapura sobre Integridade em Pesquisa (2010) [5] e outros. iii. Hoje, integridade científica, excelência em pesquisa e o potencial criativo das instituições estão dentre os principais fatores que definem competitividade em C,T\&I.

iv. A percepção de que uma contribuição significativa para a ciência é inseparável da responsabilização nas atividades de pes quisa se reflete em mudanças significativas nos critérios de julgamento sobre a qualidade e solidez de projetos e de arti gos científicos.

v. Qualidade e solidez são características interligadas, que dependem da confiabilidade dos dados e de sua fiel representação nos registros de pesquisa. 
vi. Qualidade e solidez podem ser afetadas por conflitos de interesses não revelados, que podem distorcer os registros de pesquisa e enganar a comunidade de pesquisa.

vii. No Brasil, crescentes incentivos para o desenvolvimento de projetos colaborativos, em um ambiente internacional cada vez mais competitivo, podem gerar resultados ainda mais promissores se as instituições, periódicos acadêmicos, sociedades científicas e agências de financiamento estabelecerem medidas estratégicas para promover e sustentar a responsabilização nas atividades de pesquisa e a confiança pública na ciência.

viii. A Fundação de Amparo à Pesquisa do Estado de São Paulo (FAPESP) e o ConseIho Nacional de Desenvolvimento Científico e Tecnológico (CNPq) recentemente promoveram ações pioneiras para o estabelecimento de uma cultura de responsabilização na ciência brasileira.

ix. A ciência brasileira deve ampliar seu compromisso de promover e manter uma cultura de integridade científica sintonizada com os diálogos internacionais que hoje se travam sobre RI/RCR [sigla internacional para "research integrity and responsible c onduct of research" - integridade em pesquisa e conduta responsável em pesquisa].

Como uma tentativa de abordar essas questões e contribuir com iniciativas para promover ações sobre integridade científica no Brasil, o II Encontro Brasileiro sobre Integridade em Pesquisa, Ética na Ciência e em Publicações (II BRISPE) recomenda que as instituições do país

1. incluam, promovam e divulguem orientações sobre RI/RCR e materiais informativos e didáticos sobre o tema em se us sites oficiais - recomendando-se como documentos-base a Declaração de Cingapura sobre Integridade em Pesquisa, 2010 [5]; 0 Código de Boas Práticas em Pesquisa da FAPESP, 2011 [6], as Diretivas para a Integridade da Pesquisa do CNPq, 2011 [7], e o documento "Cooperação entre Instituiç̃̃es de Pesquisa e Periódicos em Casos de Integridade em Pesquisa: Orientação do Comitê [Internacional] de Ética em Publicações" do COPE, 2012 [8];

2. incluam diretrizes sobre integridade científica em suas abordagens estratégicas para promover a excelência em pesquisa;

3. conscientizem os alunos de que o plágio é uma violação acadêmica, seja no ensino fundamental, ensino médio ou universitário. As instituições de ensino e pesquisa do país devem fornecer materiais educativos que mostrem q ue o plágio em monografias, dissertações e teses também é, além de violação acadêmica, uma prática ilegal no Brasil ;

4. proporcionem atividades educativas sobre RI/RCR entre alunos e professores para estimular a disc ussão institucional sobre as preocupações locais que devem ser trabalhadas;

5. incentivem os alunos e professores a participar de reuniões nacionais e internacionais e/ou cursos sobre RI/RCR;

6. estimulem atividades de sensibilização sobre o papel da ética em publicações e sobre a autoria acadêmica em trabalhos colaborativos, sejam eles nacionais ou internacionais;

7. ofereçam oportunidades para que estudantes e professores possam desenvolver competências linguísticas internacionais para a comunicação responsável da ciência e seus resultados para seus pares e para a sociedade; 
8. desenvolvam iniciativas, entre os estudantes de graduação e pós-graduação, para promover a noção de responsabilização nas atividades de pesquisa e a confiança pública na ciência;

9. divulguem este documento entre estudantes, pesquisadores e professores brasileiros.

Referências:

1 - Institute of Medicine \& National Research Council (2002). Integrity in Scientific Research: Creating an Environment that

Promotes Responsible Conduct. Washington, DC: National Academies Press.

2 - ESF Member Organisation Forum on Research Integrity (2010). Fostering Research Integrity in Europe. Executive Report, European Science Foundation.

3 - ICSU (2011). ICSU Strategic Plan II, 2012-2017. International Council for Science. Paris.

4 - Suresh, S (2012). Cultivating Global Science. Science, 336, 959.

5 - The Singapore Statement on Research Integrity (2010). Available at http://www.singaporestatement.org/statement.html

6 - The State of São Paulo Research Foundation (FAPESP). FAPESP's Code for Good Research Practices (2011), São Paulo. Available at http://www.fapesp.br/boaspraticas/codigo_050911.pdf

7 - Brazilian National Research Council for Scientific and Technological Development (CNPq) (2010). CNPq's Directives for

Research Integrity. Brasilia. Available at http://www.cnpq.br/web/guest/diretrizes

8 - Committee on Publication Ethics (COPE (2012). Cooperation between Research Institutions and Journals on Research

Integrity Cases: Guidance from the Committee on Publication Ethics. London. Available at http://publicationethics.org/files/Research_institutions_guidelines_final.pdf

O II BRISPE, http://www.iibrispe.coppe.ufrj.br, foi organizado pelo Instituto de Bioquímica Médica (IBqM/UFRJ) em associação com o Instituto Alberto Luiz Coimbra de Pós Graduação e Pesquisa em Engenharia (COPPE/UFRJ), tendo como colaboradores o Instituto Oswaldo Cruz (FIOCRUZ); a Universidade de São Paulo (USP) e a Pontifícia Universidade Católica doRioGrande doSul(PUCRS), alémdeoutras instituições colaboradoras. Dentre os colaboradores/patrocinadores destacam-se a Universidade Federal de São Paulo (UNIFESP), o Conselho Nacional de Desenvolvimento Científico e Tecnológico (CNPq), a Fundação de Amparo à Pesquisa do Estado de São Paulo (FAPESP), a Academia Brasileira de Ciências (ABC), a Sociedade Brasileira para o Progresso da Ciência (SBPC), o Forum de Pró- Reitores de Pós-Graduação e Pesquisa (FOPROP), o Conselho Federal da Ordem dos Advogados do Brasil (CF OAB), a OAB-Ceará, o Office of Research Integrity (ORI), os National Institutes of Health (NIH) e as All European Academies (ALLEA), dentre outros. As recomendações nesta declaração conjunta foram feitas pelos membros do Grupo de Trabalho do II BRISPE e discutidas antes, durante e após a seção sobre políticas internacionais para integridade científica/conduta responsável em pesquisa (RI/RCR), realizada em 29 de maio de 2012, na COPPE/UFRJ. Esse grupo de trabalho inclu- 
iu palestrantes convidados e membros dos comitês organizador e assessor do II BRISPE, listados a seguir:

\section{Coordenadores do Grupo de Trabalho do II BRISPE:}

Sonia Vasconcelos, PhD, Programa de Educação, Gestão e Difusão em Biociências do Instituto de Bioquímica Médica (PEGeD/IBqM/UFRJ); Martha Sorenson, PhD, Programa de Bioquímica e Biologia Celular do IBqM/UFRJ e Edson Watanabe, PhD, Programa de Engenharia Elétrica do Instituto Alberto Luiz Coimbra de Pós-Graduação e Pesquisa em Engenharia (PEE/COPPE); Diretor Acadêmico da COPPE/UFRJ.

\section{Colaboradores Nacionais:}

Adalberto Vieyra, PhD, Instituto de Biofísica Carlos Chagas Filho (IBCCF/UFRJ); Coordenador de Área (2005-2011) - Ciências Biológicas II - da Coordenação de Aperfeiçoamento de Pessoal de Nível Superior (CAPES). Membro do Comitê Organizador do I e do II BRISPE.

André Pinto, PhD, Centro Brasileiro de Pesquisas Físicas (CBPF); Vice-Presidente de Materiais da Sociedade Brasileira de

Microscopia e Microanálise (SBMM) (2009-2011). Membro do Comitê Organizador do I e do II BRISPE.

Antonio Nóbrega, PhD, Departamento de Fisiologia e Farmacologia da Universidade Federal Fluminense (UFF); Pró-Reitor de Pós- Graduação, Pesquisa e Inovação da UFF. Membro do Comitê Assessor do II BRISPE.

Carla Bonan, PhD, Faculdade de Biociências da Pontifícia Universidade Católica do Rio Grande do Sul (PUCRS); Coordenadora de

Pesquisa da Pró-Reitoria de Pós-Graduação e Pesquisa da PUCRS. Membro do Comitê Organizador do II BRISPE.

Carlos H.B. Cruz, PhD, Instituto de Física Gleb Wataghin, Universidade Estadual de Campinas (UNICAMP); Diretor Científico da

FAPESP. Membro do Comitê Assessor do II BRISPE.

Cássia Turci, PhD, Instituto de Química (IQ/UFRJ); Diretora do IQ/UFRJ. Membro do Comitê Assessor do II BRISPE.

Charles Pessanha, PhD, Programa de Políticas Públicas, Estratégias e Desenvolvimento do Instituto de Economia (PPED/UFRJ); Ex- Presidente da Associação Brasileira de Editores Científicos (ABEC). Membro do Comitê Organizador do II BRISPE.

Claude Pirmez, PhD, Laboratório de Pesquisas Médicas Multidisciplinares do Instituto Oswaldo Cruz (FIOCRUZ); Vice-Presidente de Pesquisa e Laboratórios de Referência da FIOCRUZ. Membro do Comitê Organizador do II BRISPE.

Dalton Ramos, PhD, Faculdade de Odontologia da Universidade de São Paulo (USP); Coordenador do Departamento de

Odontologia Social; Membro da Comissão Nacional de Ética em Pesquisa (CONEP). Membro do Comitê Organizador do II BRISPE.

Debora Foguel, PhD, IBqM/UFRJ; Pró-Reitora de Pós-Graduação e Pesquisa da UFRJ. Presidente da Sociedade Brasileira de

Bioquímica e Biologia Celular (SBBq) (2008-2010). Membro do Comitê Assessor do II BRISPE. Francisco Prosdocimi, PhD, IBqM/UFRJ. Membro do Comitê Organizador do II BRISPE. 
Hatisaburo Masuda, PhD, IBqM/UFRJ; Coordenador do PEGeD/IBqM/UFRJ. Membro do Comitê Organizador do I e do II BRISPE.

Jacqueline Leta, PhD, IBqM/UFRJ; Coordenadora do Laboratório de Métricas e Informação em Ciência e Tecnologia do

PEGeD/IBqM/UFRJ. Membro do Comitê Assessor do II BRISPE.

José Carlos Pinto, PhD, COPPE/UFRJ; Diretor Adjunto de Tecnologia e Inovação da COPPE/UFRJ. Membro do Comitê

Organizador do I e do II BRISPE.

José Roberto Lapa, PhD, Hospital Universitário Clementino Fraga Filho (HUCFF/UFRJ); Coordenador do Programa de Pós- Graduação em Clínica Médica da UFRJ. Membro do Comitê Organizador do II BRISPE.

Luiz Henrique L. Santos, PhD, Faculdade de Filosofia, Letras e Ciências Humanas da Universidade de São Paulo (FFLCH/USP). Coordenador de Área - Humanidades da FAPESP.

Maria D. Vargas, PhD, Instituto de Química da UFF (IQ/UFF); Coordenadora de Area - Química - da Fundação Carlos Chagas

Filho de Amparo à Pesquisa do Estado do Rio de Janeiro (FAPERJ). Membro do Comitê Assessor do II BRISPE. Mário A. C. Silva Neto, PhD, IBqM/UFRJ; Diretor do IBqM/UFRJ. Membro do Comitê Organizador do II BRISPE.

Marisa Palácios, PhD, Instituto de Saúde Coletiva (IESC/UFRJ); Coordenadora do Comitê de Ética (CEP) do IESC/UFRJ; Membro

da Diretoria da Sociedade Brasileira de Bioética (SBB). Membro do Comitê Organizador do I e do II BRISPE.

Marisa Russo Lecointre, PhD, Escola de Filosofia, Letras e Ciências Humanas da Universidade Federal de São Paulo

(EFLCH/UNIFESP). Membro do Comitê Organizador do II BRISPE.

Paulo Sérgio Beirão, PhD, Instituto de Biologia (ICB/UFMG); Diretor de Ciências Agrárias, Biológicas e da Saúde - Conselho Nacional de Desenvolvimento Científico

e Tecnológico (CNPq); Presidente da Comissão de Integridade em Pesquisa do CNPq. Membro do Comitê Assessor do II BRISPE.

Ricardo Bacelar, MA, Faculdade de Direito da Universidade de Fortaleza (UNIFOR); Corregedor-geral da Ordem dos Advogados do

Brasil - Ceará (OAB-CE). Membro do Comitê Assessor do II BRISPE.

Rosemary Shinkai, PhD, Faculdade de Odontologia da PUCRS; Coordenadora da Divisão de Projetos da Pró-Reitoria de Pós- Graduação e Pesquisa da PUCRS. Membro do COPE. Membro do Comitê Organizador do II BRISPE.

Stevens Rehen, PhD, Instituto de Ciências Biológicas (ICB/UFRJ); Coordenador do Laboratório Nacional de Células Tronco

Embrionárias (LANCE). Membro do Comitê Assessor do II BRISPE.

\section{Colaboradores Internacionais:}

Ana Marusic, PhD, Universidade Split, Croácia; Presidente do Council of Science Editors (CSE) (2007-2008) e da World Association of Medical Editors (WAME) (2002-2003). Membro do Comitê Assessor do II BRISPE. 
Debra Mathews, PhD, Universidade de Johns Hopkins; Diretora Assistente de Programas Científicos no Instituto Berman de

Bioética da Universidade de Johns Hopkins, Estados Unidos.

Donald McCabe, PhD, Rutgers Business School-Newark and New Brunswick, Rutgers, Universidade Estadual de New Jersey, Estados Unidos. Membro do Comitê Assessor do II BRISPE.

Elizabeth Wager, PhD, Presidente do Comitê [Internacional] de Ética em Publicações - COPE (2009-2012), Reino Unido. Membro do Comitê Assessor do II BRISPE.

Harold Garner, PhD, Instituto de Bioinformática da Virginia; Diretor da Divisão Médica de Informática e Sistemas da Universidade

Estadual e Instituto Politécnico da Virginia, Estados Unidos.

Ivan Oransky, MD, Editor Executivo do Reuters Health, Estados Unidos; Cocriador do Retraction Watch. Membro do Comitê

Assessor do II BRISPE.

Jane Robbins, PhD, Centro de Empreendedorismo McGuire, Universidade do Arizona, Estados Unidos.

Linda Nielsen, PhD, Universidade de Copenhagen; Copresidente do Painel Europeu sobre Ética na Ciência e em Novas

Tecnologias e do Comitê Intergovernamental de Bioética para a UNESCO, Dinamarca. Membro do Comitê Assessor do II BRISPE.

Miguel Roig, PhD, Departamento de Psicologia da Universidade St. John's, Estados Unidos. Membro do Comitê Assessor do II BRISPE.

Nicholas Steneck, PhD, Universidade de Michigan; Diretor do Programa de Ética e Integridade em Pesquisa do Instituto Michigan para Pesquisas Clínicas e em Saúde (MICHR), Estados Unidos; Copresidente da Primeira e da Segunda Conferências Mundiais sobre Integridade Científica, 2007 e 2010. Membro do Comitê Assessor do II BRISPE.

Rüdiger Klein, PhD, Diretor Executivo das All European Academies (ALLEA), Federação Europeia de Academias Nacionais de

Ciências e Humanidades, Holanda.

Sabine Kleinert, PhD, Editora Executiva do The Lancet; Copresidente do COPE (2009-2012), Reino Unido; Copresidente da

Terceira Conferência Mundial sobre Integridade Científica, 2013. Membro do Comitê Assessor do II BRISPE.

Susan Zimmerman, BCL, LLB, Diretora Executiva da Secretaria Geral sobre Ética em Pesquisa do Governo Canadense, Canadá. Membro do Comitê Assessor do II BRISPE. 